\title{
The Firing Method for Naval Gun to Fire on Shore Based on UAV
}

\author{
Qiang $\mathrm{Gai}^{1, \mathrm{a}}$, Jiang $\mathrm{Hu}^{1, \mathrm{~b}}$ \\ ${ }^{1}$ Dept. of Equipment System, Dalian Naval Academy, China \\ axiangjiangzhishui@163.com, jiantingxueyuan@163.com
}

\begin{abstract}
.
The firing method for naval gun to fire on shore based on UAV (Unmanned Aerial Vehicle) extends observation range of warship, and it is a remote firing method that can be widely used. At first, this paper describes the flow for naval gun to reconnaissance and firing correction based on UAV. And then by using principle of collinear structure image, the target's location method based on UAV is put forward. In the end, the computational model of target's relative coordinates is established. The research result provides reference for naval gun to attack shore target which is sightless, at the same time it holds important theoretic significance and practical value.
\end{abstract}

Keywords: UAV; naval gun; relative coordinates

\section{Introduction}

With the advantages of fast emission velocity and continuous attack ability, naval gun is usually used as oppressive weapon during firing on shore [1]. The surroundings of the shore target are evidently different from that at sea. When naval gun attacking the shore target, due to obstruct caused by terrain, performance limitation of shipborne observation facility and so on, it may be difficult to observe the target and impact point effectively.

If UAV which equipped with TV observation equipment and integrated navigation system is used to execute observation and firing correction, it can not only determinates location of target accurately, but also maneuvers easily, 
evacuates rapidly and avoids fire attacking from enemy [2]. So the firing method for naval gun to fire on shore based on UAV is one of effective safeguard method.

In the process of naval gun firing on shore based on UAV, the important problem is to set up the calculation model of relative coordinate. Therefore, this paper describes the flow for naval gun to reconnaissance and firing correction based on UAV firstly, and then focuses on the calculation model of relative coordinate.

\section{The flow of reconnaissance and firing correction}

The implementation method for UAV to reconnaissance and firing correction is usually as follows: During firing on shore, UAV is assigned to approach target in the air. According to appointed connective method and location method, UAV observes target's coordinate and situation of burst point, and it offers the information of location, firing correction parameters and strike effect to ship.

During naval gun firing on shore, the flow for naval gun to reconnaissance and firing correction based on UAV is as following:

(1)Implementing combat preparation. After receiving the commission of firing on shore, director organizes related staff to implement firing preparation. And ship position $\left(\lambda_{w}, \phi_{w}\right)$ is determined by shipborne navigation system [3].

(2)Indicating reconnaissance area. Director explains the target information, including properties and terrain, to UAV. And the reconnaissance area and airway of UAV are indicated.

(3)Measuring target's position. According to the order of director, UAV take off from ship. When observation equipment of UAV discovers the shore target, then UAV turns into automatically tracking and measuring state. The schematic drawing of observing target by UAV is as Fig.1. 


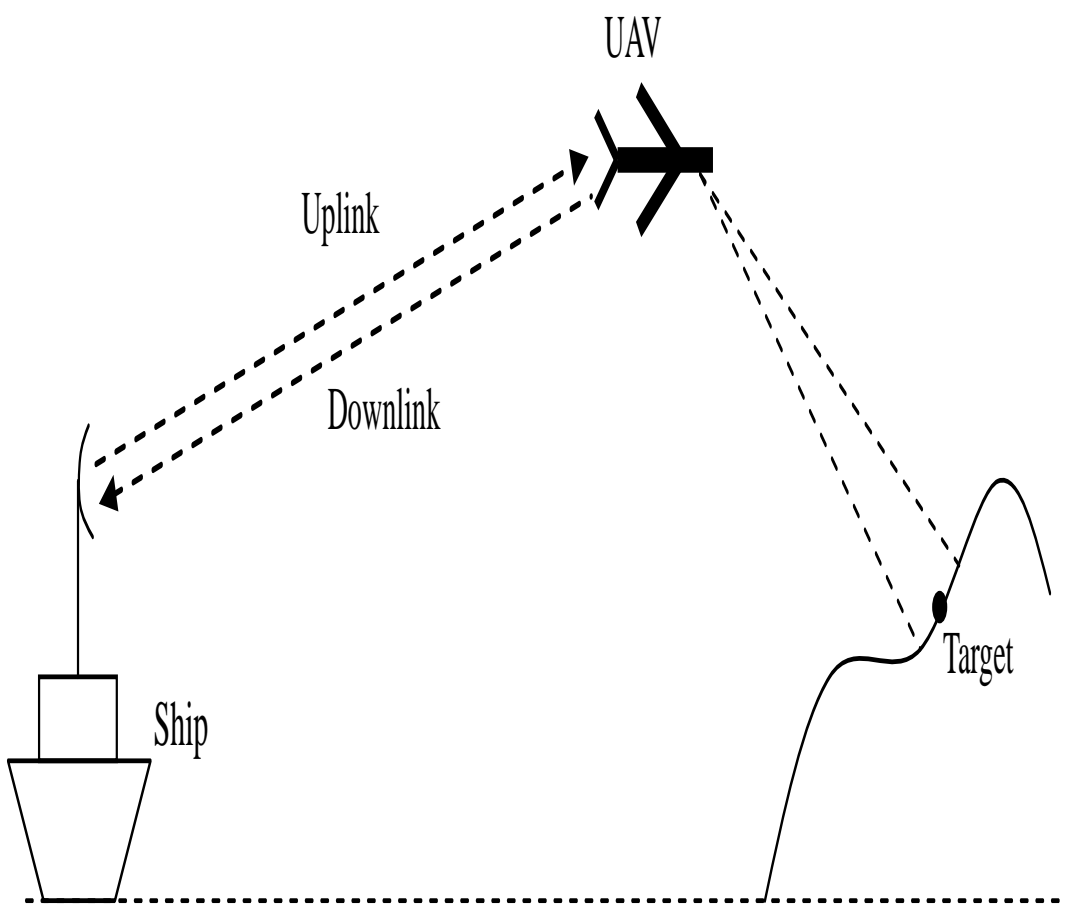

Fig.1 The schematic drawing of observing target by UAV

(4)Calculating firing data. By utilizing radio communication or data link, UAV transfers target's location information to fire control system of naval gun. After correcting the influences of ballistic and meteorological factors, fire control system works out firing data and drive naval gun to aim target.

(5)Observing impact deviation. If the firing requirements are satisfied, fire control system make naval gun to fire. Then director order UAV to start firing observation. After UAV has detected image of target and impact, it studies and determines the information, and give feedback to fire control system. The fire control system calculates firing correction data, and implements firing correction.

(6)Evacuating from combat area. If naval gun has completed firing task, UAV will evacuate from combat area immediately to avoid damage of enemy.

When the hovering and observing position of UAV is considered, the basic requirement is that the target and impact point will be observed accurately, and the safe of UAV can be ensured. 


\section{The computational model of relative coordinates}

So long as the coordinate of Warship and UAV can be confirmed, we may calculate the target's relative coordinates.

Determining Position of Warship. Warship need navigation system to locate its position when firing on shore, therefore, location accuracy of navigation system is required to reach high level. At present, the main navigation system is Loran-c and satellite positioning system. The satellite positioning system has advantages of high precision and so on, and it is the important positioning system in future naval battle [4].

The typical satellite positioning system is GPS, and GPS hold the merits of all-weather, high coverage and continuity. The positioning accuracy of GPS is about $10 \mathrm{~m}$, and it meets accuracy requirement for naval gun to fire on shore completely [5].

When the GPS is used alone, the number of satellite whose signal can be received is just about three or four, and it may make the location error bigger. If the integrated location of GPS and GLONASS is applied, the number of satellite whose signal can be received is certain to increase, and it may remarkably raise the location accuracy.

Target's Location Model Based on Principle of Collinear Structure Image. According to imaging principle of matrix CCD camera which is equipped by UAV, we can know that, the ground target, projection center and the target's image points on camera target plane are always on one line. This is the famous principle of collinear structure image in photogrammetry [6].

Based on the principle of collinear structure image, after information including target's coordinate on camera target plane, three-dimensional coordinate of UAV and focal length, spatial attitude of camera are acquired, two-dimensional coordinate of target can be determined.

After the rectangular space coordinate $\left(X_{s}, Y_{s}\right)$ and hovering height $H_{s}$ of UAV are accurately measured by airborne navigation system, in accordance with 
principle of collinear structure image, the calculation model of target's rectangular space coordinate $\left(U_{m}, V_{m}\right)$ is as follows:

$$
\left\{\begin{array}{l}
U_{m}=X_{s}-\left(H_{s}-Z_{m}\right) \frac{a_{1} x_{r}-a_{2} f+a_{3} y_{r}}{b_{1} x_{r}-b_{2} f+b_{3} y_{r}} K \\
V_{m}=Y_{s}-\left(H_{s}-Z_{m}\right) \frac{c_{1} X_{r}-c_{2} f+c_{3} y_{r}}{b_{1} x_{r}-b_{2} f+b_{3} y_{r}} K
\end{array}\right.
$$

In above formula, $f$ means camera's focal length; $\left(x_{r}, y_{r}\right)$ means the target's coordinate on camera target plane; $K$ means the proportional coefficient between camera image and monitor image; $a_{1}, a_{2}, a_{3}, b_{1}, b_{2}, b_{3}, c_{1}, c_{2}, c_{3}$ mean the attitude angle function of UAV.

Meanwhile, target elevation $Z_{m}$ can be obtained by DEM (Digital Elevation Model). In the light of target's rectangular space coordinate $\left(U_{m}, V_{m}, Z_{m}\right)$, if we use the conversion formula between rectangular space coordinate and geodetic coordinate, we may get the target's latitude and longitude coordinate $\left(\lambda_{m}, \phi_{m}\right)$.

When the principle of collinear structure image is utilized for confirming target's position, several kinds of errors are to be considered, including the locating error of airborne platform, attitude measuring error and the extracting error of image point.

With the development of navigation and detecting technology, new technology such as synthetic aperture radar and image matching can be used by UAV to locating target [7]. And it will satisfy the high accuracy demand on target's coordinate when naval gun firing on shore [8].

Calculation of Target's Relative Coordinate. After the ship's coordinate $\left(\lambda_{w}, \phi_{w}\right)$ and target's coordinate $\left(\lambda_{m}, \phi_{m}\right)$ are obtained, if we want to 
conveniently solve target's relative distance $d$ and relative azimuth $f$, Gauss projection positive calculation formula can be used [9].

According to Gauss projection positive calculation formula, the Gauss plane rectangular coordinate $\left(x_{m}, y_{m}\right)$ of target and Gauss plane rectangular coordinate $\left(x_{w}, y_{w}\right)$ of ship are calculated respectively.

Then the relative rectangular coordinate are as follows:

$$
\left\{\begin{array}{l}
\Delta x=x_{m}-x_{r} \\
\Delta y=y_{m}-y_{r}
\end{array}\right.
$$

Therefore, the formula to calculate target's relative distance $d$ is as follows:

$$
d=\sqrt{(\Delta x)^{2}+(\Delta y)^{2}}
$$

The formula to calculate target's relative azimuth $f$ is as follows:

$$
f= \begin{cases}0 & \Delta x=0 \text { 且 } \Delta y \geq 0 \\ \pi & \Delta x=0 \text { 且 } \Delta y \leq 0 \\ \frac{\pi}{2}-\operatorname{tg}^{-1}\left(\frac{\Delta y}{\Delta x}\right) & \Delta x>0 \\ \frac{3 \pi}{2}-\operatorname{tg}^{-1}\left(\frac{\Delta y}{\Delta x}\right) & \Delta x<0\end{cases}
$$

\section{Conclusion}


When UAV is used as observation support equipment for naval gun to fire on shore, the observation distance may reach the maximum range of naval gun. It not only extends the observation scope of warship, but also is beneficial for naval gun to full play operational effectiveness. The firing method for naval gun to fire on shore based on UAV is a remote firing method that can be widely used.

\section{Acknowledgements}

The paper is supported by Research Development Fund of Dalian Naval Academy.

\section{References}

[1] C.H. ZHAO and J. BAI: Shipboard Weapons, Vol. 21(2002) No.2, p.33.

[2] M. ATHANS: IEEE Transactions on Automatic Control, Vol. 32(1987) No.4, p.286.

[3] Y.WANG: Research and Realization of the Algorithm on the Ship Track Keeping (Harbin Engineering University, China 2008).

[4] X.T. WANG, Electric Navigation Instrument (Dalian naval academy, China 1994).

[5] S.H. ZUO: Geomatics and Spatial Information Technology, Vol. 30(2007) No.4, p.42.

[6] Z.Z.WANG: Principles of photogrammetry (Surveying and Mapping Press, Beijing, China 1999).

[7] B. KARLIK:Mathematical and Computational Applications, Vol. 8(2003) No.1, p.181.

[8] M.Y. LIN: Radar Signal Theory (National Defense Industry Press, China 2007).

[9] S.Q. XU: Geodetic Surveying (Wuhan Technical University of Surveying and Mapping, China 1996). 